\title{
Leistungsbewertung und -optimierung in der manuellen Kommissionierung
}

\author{
Performance Assessment and Optimization in Manual Order Picking
}

\author{
Matthew Stinson \\ Karl-Heinz Wehking \\ Institut für Fördertechnik und Logistik (IFT), Universität Stuttgart
}

$\mathbf{I}_{\mathrm{u}}^{\mathrm{n}}$ n der Auftragszusammenstellung spielt der Mensch u. a. aufgrund seiner hohen Flexibilität bezüglich der Aufgabeneinteilung und der Fähigkeit, unterschiedlich dimensionierte Objekte leicht handzuhaben eine wesentliche Rolle. Um die Leistungen der Mitarbeiter individuell beurteilen zu können, ist die Berücksichtigung heterogener Arbeitsinhalte notwendig. Beispielsweise ist anzunehmen, dass Kommissionieraufträge mit relativ schweren Artikeln mehr Zeit in Anspruch nehmen als Aufträge mit leichteren Artikeln. Ebenso nimmt der Kommissionieraufwand zu, wenn mehr Auftragspositionen anzufahren sind und wenn größere Distanzen zurückgelegt werden müssen. Durch die Quantifizierung solcher Leistungseinflussfaktoren auf Mitarbeiterebene können individuelle Leistungsprofile erstellt werden, aus denen ganzheitliche Leistungsbeurteilungen sowie Optimierungen in der Personaleinsatzplanung und Kommissionierauftragssteuerung hervorgehen.

[Schlüsselwörter: Manuelle Kommissionierung, Person-zurWare Personaleinsatzplanung, Auftragssteuerung]

I n order fulfillment, manual labor remains invaluable due in part to humans' flexibility in respect to task assignment and their ability to handle objects of various sizes. In order to evaluate individual workers' performance, heterogeneous work contents must be considered. For example, it is assumed that picking orders containing relatively heavy items require more time to fulfill than picking orders with lighter items. By the same token, the required picking effort is increased as the number of order lines and the required walking distance increase. Through the quantification of such performancerelevant input factors on the employee level, individual performance profiles can be created. These profiles can then be used as a basis for holistic performance evaluations as well as for optimizations in personnel planning and picking order assignment.

[Keywords: manual picking, person-to-stock, personnel planning, order assignment]

\section{Ausgangssituation}

Aktuelle Forschungsergebnisse bestätigen die Rolle des Menschen als wesentlicher operativer leistungsträger in der Distributionslogistik. U. a. sein Problemlösungsund Kommunikationsvermögen, seine Anpassungsfähigkeit sowie die mit ihm verbundenen geringen Investitionskosten liegen dem grundsätzlich geringen Automatisierungsgrad in der Distributionslogistik zugrunde [Cro07; Deu2009; Hom10; Spa09]. Zur Planung, Steuerung und Optimierung von automatischen Kommissioniersystemen wurden einige Berechnungs- und Bewertungsmethoden entwickelt [Gro83; Gud05]. Jedoch existiert trotz des typisch hohen Kostenanteils der manuellen Kommissionierung in Bezug auf die Gesamtbetriebskosten in der Distribution keine Methode zur ganzheitlichen Leistungsbewertung in manuellen Kommissioniersystemen. Einschlägige Berechnungsmethoden kommen i. d. R. lediglich zur Dimensionierung von Arbeitssystemen in der Planungsphase zum Einsatz [Gud05].

Gegenwärtige Leistungsbewertungsmethoden in der manuellen Kommissionierung sind häufig weder in der Lage, individuelle Leistungsbewertungen noch eine mehrdimensionale Beurteilung der Kommissionierleistung zu gewährleisten. Zur Leistungsbewertung der Mitarbeiter wird häufig die Gesamtleistung der Belegschaft betrachtet, wobei die individuelle Leistungsbewertung ggf. nur in Hinsicht auf z. B. Picks/Std. oder Kommissionieraufträge/Std. vorgenommen wird [VDI94]. Eine Leistungsbewertung, die lediglich auf der entnommenen Menge beruht, ist nicht ausreichend zur Beurteilung der realen Kommissionierleistung, da wesentliche Einflussfaktoren auf die Kommissionierleistung nicht in Betracht gezogen werden [GaW10]. Somit werden die Voraussetzungen zur Optimierung durch die Berücksichtigung individueller Stärken und Schwächen der Kommissionierer weder bei der Personaleinsatzplanung noch bei Schulungen oder bei der Entlohnung erfüllt. Im Rahmen des Forschungsprojekts „EfKom“ (Bewertung \& Optimierung der Effizienz manueller Tätigkeiten in der Kommissionierung / AGFVorhaben 17236 N werden Ansätze zur Behebung der oben erläuterten Problematik in der Praxis erprobt. Der Kern dieses Beitrags beruht hauptsächlich auf der für das 
Projekt entwickelten Methodik sowie die aus der praktischen Anwendung gewonnenen Erkenntnisse.

\section{BESTIMMUNG DER LEISTUNGSEINFLUSSFAKTOREN}

Zur Leistungsmessung werden aktuell hauptsächlich mengenbezogene Kennzahlen herangezogen, z. B. Kommissionieraufträge oder Entnahmen pro Stunde, bezogen auf das gesamte Kommissioniersystem. In den letzten Jahren wurden zusätzliche Leistungsaspekte in der manuellen Kommissionierung untersucht [Cro07; Deu09; Hom10; Sch09; Spa09]. Eine Studie der TU München hat bereits u. a. folgende Leistungseinflussfaktoren auf die Kommissionierleistung identifiziert:

- die Heterogenität der Aufträge,

- die Heterogenität des Sortiments und

- den Leistungsumfang.

Dementgegen hat die TU Dresden im Rahmen eines Forschungsprojekts folgende Leistungseinflussfaktoren untersucht [Sch09]:

- die Kommissionierzeit und Pickmenge,

- die Kommissionierzeitschwankungen,

- den Leistungsgrad nach Alter und

- den Leistungsgrad nach Betriebszugehörigkeit.

Eine eindeutige Zuordnung zwischen einzelnen Mitarbeitern und Leistungskennzahlen stellt im Projekt EfKom eine Weiterentwicklung dar. Ergänzend hierzu bietet sich die Betrachtung von zusätzlichen Faktoren, die die Kommissionierleistung maßgeblich beeinflussen. Hierzu werden im Rahmen des Forschungsprojekts neben der Anzahl Kommissionieraufträge, -positionen und Entnahmen auch folgende Leistungseinflussfaktoren untersucht:

- $\quad$ Die mit einem Kommissionierauftrag verbundene Wegstrecke,

- $\quad$ Die Massen der zu kommissionierenden Artikel,

- Die Volumina der zu kommissionierenden Artikel.

Hierzu werden folgende Thesen aufgestellt, deren jeweilige Aussagekraft im späteren Projektverlauf statistisch geprüft werden sollen:
These 1:

Eine kausale, positive Korrelation besteht zwischen der Anzahl der in einem Kommissionierauftrag enthaltenen Entnahmen und der Kommissionierauftragsbearbeitungszeit. Begründung hierfür ist eine Erhöhung der Greif- und Totzeiten durch u. a. zusätzliche Handhabung (z. B. Arrangieren der Kartoninhalte) und Prüfvorgänge (z. B. Scannen jedes entnommenen Artikels und Nachzählen).

\section{These 2:}

Zwischen der Anzahl der in einem Kommissionierauftrag enthaltenen Kommissionierpositionen und der Kommissionierauftragsbearbeitungszeit existiert eine positive, kausale Korrelation. Dieses ist damit zu begründen, dass bei jeder Kommissionierposition zusätzliche Totzeit anfällt und dass die Laufgeschwindigkeiten durch zusätzliche Brems- und Beschleunigungsvorgänge geringer werden.

These 3:

Eine kausale, positive Korrelation besteht zwischen der für die Bearbeitung eines Kommissionierauftrags zurückzulegende Wegstrecke und der Kommissionierauftragsbearbeitungszeit. Der Grund hierfür ist die Wegzeit.

These 4:

Die Kommissionierauftragsmasse und die Kommissionierauftragsbearbeitungszeit stehen in einer positiven, kausalen Korrelation. Dieses lässt sich mit der reduzierten Gehgeschwindigkeit sowie dem erhöhten Handhabungsaufwand erklären.

Die Zielsetzung in der Berücksichtigung von kommissionierauftragsspezifischen Einflussfaktoren besteht darin, eine Vergleichsbasis auf Kommissionierauftragsebene zu schaffen. Diese Betrachtungsweise ist aus praktischer Sicht günstig, da Teilkommissionierauftragszeiten, z. B. Basis-, Geh-, Such-, und Greifzeit häufig nicht erfasst werden. Die Berücksichtigung weiterer Leistungseinflussfaktoren, die sich nicht unmittelbar auf die Kommissionieraufträge beziehen, bspw. das Qualifikationsniveau des Mitarbeiters, der aktuelle Auslastungsgrad des Kommissioniersystems und die Uhrzeit ist durchaus denkbar.

Zur Untersuchung kausaler Zusammenhänge ist zusätzlich zu überprüfen, ob Abhängigkeiten zwischen den Leistungseinflussfaktoren vorhanden sind. Hierzu ist die Analyse einschlägiger eindimensionalen Verteilungen erforderlich. Bei einer homogenen Massenverteilung der Artikel sollte insbesondere auf eine kausale Korrelation zwischen der Anzahl in einem Kommissionierauftrag enthaltenen Entnahmen und der Kommissionierauftragsmasse geprüft werden. 


\section{KENNZAHLENERHEBUNG}

Zur möglichst ganzheitlichen Quantifizierung der Kommissionierleistung werden verschiedene Kennwerte herangezogen. Hierbei bieten sich die Ermittlung folgender Kennzahlen an:

- Kommissionieraufträge pro Stunde (netto ${ }^{1}$ ) [Stk/h]

- Kommissionierpositionen pro Stunde (netto) [Stk/h]

- $\quad$ Entnahmen pro Stunde (netto) $[\mathrm{Stk} / \mathrm{h}]$

- Gehgeschwindigkeit pro Kommissionierauftrag ${ }^{2}$ [m/sec]

- Kommissionierte Masse pro Stunde (netto) $[\mathrm{kg} / \mathrm{h}]$

- Kommissionierauftragsdurchlaufzeit [h/Stk]

- Qualitätsgrad [\%] ${ }^{3}$ [VDI94]

Neben den zeitmengenbezogenen Kennzahlen stellt die Ermittlung von auftragsbezogenen Kennwerten eine Voraussetzung zur Optimierung der Kommissionierauftragssteuerung. Ebenso stellt die auftragsbezogene Betrachtungsweise eine Voraussetzung zur individuellen Leistungsbewertung in Hinsicht auf die zuvor genannten Leistungseinflussfaktoren.

Bei der Erhebung der Kennzahlen ist auf die Qualität der Daten zu achten. Hier sollen Faktoren wie die Anzahl der Messpunkte, die Präzision der Messungen und mögliche Datenfälschungsquellen untersucht werden. Zusätzlich sind Plausibilitätsprüfungen durchzuführen sowie die Identifizierung und ggf. der Ausschluss von Ausreißern vorzunehmen. Im betrachteten Forschungsprojekt stellt die Datenerhebung und Kennzahlenermittlung einen einmaligen Aufwand dar und ist deshalb als Momentaufnahme einzustufen, deren Zeithorizont i. d. R. mehrere

1 Die Netto-Kommissionierzeit entspricht ausschließlich der während der Kommissionierauftragsbearbeitung vergangenen Zeit.

${ }^{2}$ Um die Gehgeschwindigkeiten ermitteln zu können, ist eine Berechnung der tatsächlich gelaufenen Kommissionierwege erforderlich. Am IFT wird hierzu eine Software eingesetzt, welche anhand des Lagerlayouts, dessen Dimensionen und der eingesetzten Wegstrategie die theoretischen Wegstrecken der Kommissionieraufträge zurückgibt.

${ }^{3}$ Hierbei kann der Qualitätsgrad dem Verhältnis der Anzahl der nicht reklamierten Kommissionieraufträge zur Anzahl der reklamierten Kommissionieraufträge entsprechen, damit die reale Kommissionierleistung nicht durch fehlerhaftes Kommissionieren erhöht wird. Alternativ können an dieser Stelle auch kleinere, ggf. interne Qualitätskreise herangezogen werden.
Monate umfasst. Eine Datenerhebung und Kennzahlenermittlung in Echtzeit ist im Rahmen des Forschungsprojekts nicht vorgesehen.

\section{GANZHEITLICHE LEISTUNGSBEWERTUNG IN DER PERSON-ZUR-WARE-KOMMISSIONIERUNG}

Um eine möglichst ganzheitliche Leistungsbeurteilung auf der Mitarbeiterebene gewährleisten zu können, wird im Laufe des Projekts eine Betrachtung jedes Mitarbeiters in Hinsicht auf die vier aufgeführten Leistungseinflussfaktoren vorgenommen. In Abbildung 1 werden einschlägige Daten aus einem manuellen Kommissionierlager aufgeführt.
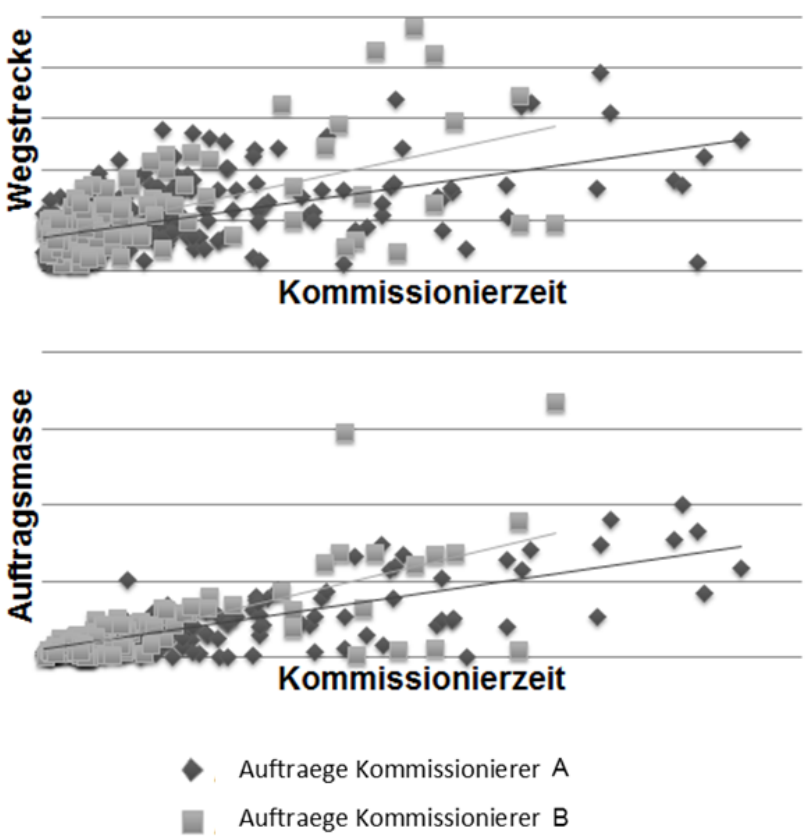

Abbildung 1. Teil-Leistungsprofile als zweidimensionale Verteilungen dargestellt

Hier werden die Leistungseinflussfaktoren „Wegstrecke“ und „Auftragsmasse“ jeweils ins Verhältnis zur Kommissionierzeit auf Kommissionierauftragsbasis pro Mitarbeiter aufgestellt. Dabei repräsentiert jeder Messpunkt einen Kommissionierauftrag, dessen X-/Y-Koordinaten der erfassten Kommissionierzeit bzw. der Wegstrecke oder Auftragsmasse entsprechen. Anhand der eingezeichneten Regressionsgeraden in beiden Diagrammen ist ein positiver Zusammenhang festzustellen, wobei die X-Variablen (Kommissionierzeit) als teilweise abhängig von den Y-Variablen verstanden werden. Die Eigenschaften der jeweiligen Punktwolken können anhand der Korrelationsrechnung beschrieben werden mit bspw. dem Korrelationskoeffizient, um die Richtung und Stärke des linearen Zusammenhangs zwischen den X- und YVariablen quantitativ auszudrücken. 
Es ergibt sich bspw. für Mitarbeiter A im unteren Diagramm ein Korrelationskoeffizient von 0,47. Dieser Wert entspricht einer positiven, mittelstarken Korrelation. Es wird angenommen, dass diese Korrelation auch kausal ist, insofern dass die X-Variablen (Kommissionierzeit) von den Y-Variablen (Auftragsmasse) teilweise abhängen. Diese Ausgleichsgeraden können wiederum als „Leistungsgeraden“ verstanden werden, da sie erwartete Arbeitsmengen bezogen auf Zeiteinheiten wiedergeben. Andere Ausgleichskurvenformen, bspw. die exponentiale Ausgleichskurve, bieten auch eine gute Annäherung an die betrachteten Messpunkte, jedoch bietet die lineare Betrachtungsweise aufgrund ihrer ebenfalls guten Annäherung an die erfassten Messpunkte sowie durch ihre Einfachheit eine gute Prognosefähigkeit bzw. eine überlegene Praxistauglichkeit.

Alle entsprechenden zweidimensionalen Verteilungen weisen die erwarteten positiven Steigungen auf (siehe Thesen 3 und 4) abgesehen von vereinzelten Fällen, in denen nur sehr kleine Stichproben pro Mitarbeiter zur Verfügung standen und entsprechend nicht berücksichtigt wurden. Es ist anzumerken, dass die Regressionsgeraden der jeweiligen Mitarbeiter unterschiedliche Anfangspunkte an der Y-Achse sowie unterschiedliche Steigungen besitzen. Diese Beobachtung impliziert unterschiedliches Verhalten der Mitarbeiter in Hinsicht auf die betrachteten Leistungseinflussgrößen.

Auf Basis der Analyse der zweidimensionalen Verteilungen (jeweils das Verhältnis Wegstrecke, Auftragsmasse, Auftragsvolumen, Anzahl Kommissionierpositionen und Anzahl Entnahmen) können entsprechende Mitarbeiterleistungsprofile erstellt werden. Diese Leistungsprofile können anhand der jeweiligen Ausgleichsgeradenformeln quantitativ dargestellt werden. In Abbildung 2 werden die erwarteten Kommissionierzeiten der jeweiligen Mitarbeiter in Abhängigkeit von der Anzahl Kommissionierpositionen zwischen 1-50 dargestellt.

Die grauen Felder in der Abbildung enthalten die geringsten erwarteten Kommissionierzeiten bei einer vorgegebenen Anzahl Kommissionierpositionen. In Abbildung 2 ist eine Überlappung der Leistungsgeraden Mitarbeiter 7 und 10 bei der Anzahl acht Kommissionierpositionen zu erkennen. Das heißt, dass Mitarbeiter 7 erfahrungsgemäß Aufträge mit 1-7 Kommissionierpositionen am schnellsten abarbeitet. Mitarbeiter 10 benötigt wiederum ab 8 Kommissionierpositionen am wenigsten Zeit für seine Kommissionieraufträge.

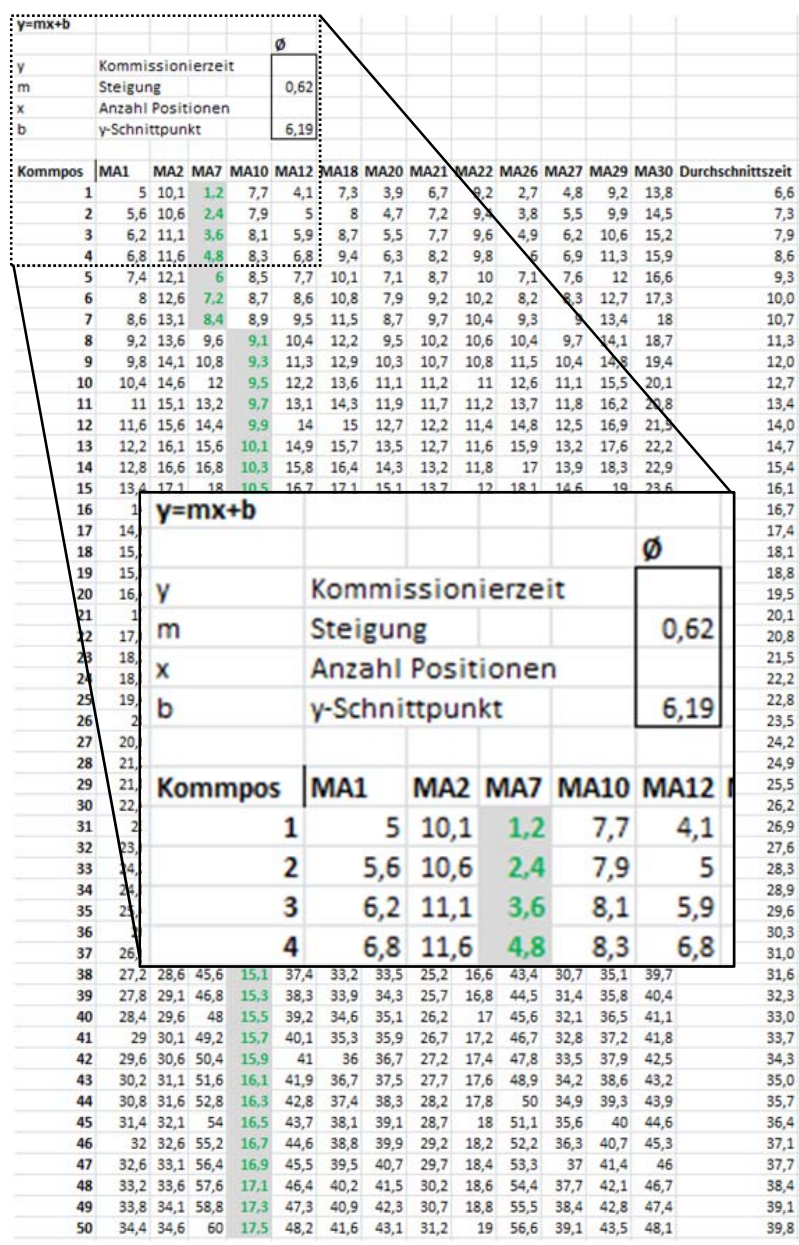

Abbildung 2. Quantifizierung der Leistungsgeraden mehrerer Mitarbeiter in Hinsicht auf die Anzahl der Kommissionierpositionen

Ausgehend von den oben dargestellten erwarteten Kommissionierzeiten können auch Mitarbeiter-Ranglisten gebildet werden. Diese können der durchschnittlichen Abweichung über oder unter den durchschnittlichen erwarteten Kommissionierzeiten der jeweiligen Mitarbeiter bei den jeweiligen Anzahlen von Kommissionierpositionen zugrunde liegen. Wenn die jeweils kürzesten Kommissionierzeiten nicht unterschiedlich gewichtet werden, dann ergibt sich anhand der Regressionsgeraden folgende Mitarbeiter-Rangliste: 


\begin{tabular}{c|cc} 
Rang & $\begin{array}{c}\text { Durchschnittliche prozentuale } \\
\text { Abweichung } \\
\text { unter Ø Kommissionierzeit }\end{array}$ & Mitarbeiter \\
\hline 1 & 40,7 & MA10 \\
2 & 34,9 & MA22 \\
3 & 16,3 & MA21 \\
4 & 13,4 & MA1 \\
5 & 4,7 & MA27 \\
6 & 3,2 & MA2 \\
7 & $-1,2$ & MA20 \\
8 & $-4,9$ & MA18 \\
9 & $-11,4$ & MA12 \\
10 & $-12,2$ & MA29 \\
11 & $-24,9$ & MA26 \\
12 & $-28,6$ & MA7 \\
13 & $-29,9$ & MA30
\end{tabular}

Abbildung 3. Mitarbeiter-Ranglistenbildung in Hinsicht auf den Leistungseinflussfaktor „Anzahl Kommissionierpositionen “

Die Werte in Abbildung 3 stellen die durchschnittlichen prozentualen Abweichungen von der mittleren erwarteten Kommissionierzeit der jeweiligen Mitarbeiter dar für 1-50 Kommissionierpositionen pro Kommissionierauftrag. Dementsprechend sind positive und negative Werte als überdurchschnittlich bzw. unterdurchschnittlich zu verstehen. Im Forschungsprojekt EfKom wird für jeden Einflussfaktor kommissioniersystemweise eine Ranglistenbildung durchgeführt, um die unterschiedlichen Stärken und Schwächen der Mitarbeiter zu identifizieren, zu quantifizieren und gegenüberzustellen. Die Ergebnisse stellen einerseits eine Vergleichsbasis dar, anderseits können sie als Grundlage zur optimierten Personaleinsatzplanung und Auftragssteuerung herangezogen werden. Die letzteren zwei Aspekte werden im Folgenden erläutert.

\section{OPtimierte PersonaleinsatzPlanung}

Anbieter von Systemen vorbestimmter Zeiten, z. B. MTM (Methods-Time-Measurement) und REFA (Verband für Arbeitsgestaltung, Betriebsorganisation und Unternehmensentwicklung) bieten mit ihren Produkten die Möglichkeit, statistisch abgesicherte Soll-Prozesszeiten festzulegen. Allerdings gehen die gängigen Systeme (z. B. MTM-1 und MTM-UAS) von einem eingearbeiteten, durchschnittlich veranlagten Mitarbeiter aus. Auf Basis der bereits ermittelten mitarbeiter- und leistungseinflussfaktorspezifischen Kommissionierleistungsbeurteilung sind genauere Kommissionierzeitprognosen möglich: Die zusätzliche Berücksichtigung von mitarbeiterspezifischen Leistungswerten bei der Personaleinsatzplanung bietet den Vorteil, Bearbeitungszeitprognosen auch in Abhängigkeit von den bearbeitenden Mitarbeitern zu ermitteln.
Anhand von kommissionierauftrags- und mitarbeiterspezifischen Prozesszeiten können auch teamspezifische Prognosewerte ermittelt werden. Im späteren Projektverlauf wird ein Prognoseverfahren entwickelt, welches einerseits einen Bezug zu den objektiven, statistisch abgesicherten Soll-Prozesszeiten nach MTM-AUS herstellt, andererseits aber die Genauigkeit der Prognosen durch individuelle $\mathrm{Zu}$ - und Abschläge erhöht. Hierbei ist auf die Korrelationskoeffizienten zu achten: Eine Leistungsgerade mit einem höheren Korrelationskoeffizienten sollte ein besserer Indikator der Kommissionierzeit sein. Auf Basis der genaueren Prognosen soll die Häufigkeit sowie das Ausmaß von Engpässen und Überkapazitäten im Kommissionierpersonal reduziert werden können.

\section{Optimierung Der Auftragssteuerung}

In der manuellen Kommissionierung sind sowohl ergonomische Mängel als auch einseitige Arbeitsbelastung kritisch einzustufen: Sie stellen einen wirtschaftlichen Nachteil und eine unnötige Zusatzbelastung für die Mitarbeiter dar. Diese Problematik soll durch entsprechende Prozessanalysen und Anpassungen bzw. eine optimierte Auftragssteuerung im Rahmen des Projekts im praktischen Umfeld verbessert werden. Die Zuordnung der in der manuellen Kommissionierung typischerweise heterogenen Kommissionieraufträge zu den geeigneten Mitarbeitern anhand von Kommissionierauftragseigenschaften bzw. individuellen Stärken und Schwächen ist aufgrund fehlender Hilfsmittel als schwierig einzustufen. Da bei einer Ad-hoc- oder willkürlichen Zuordnung von Kommissionieraufträgen die individuellen Stärken und Schwächen unberücksichtigt bleiben, ist ein optimaler Personalressourceneinsatz nicht möglich.

Ausgehend von den Mitarbeiterleistungsprofilen ist eine strategische Kommissionierauftragssteuerung denkbar. Hierzu wird im Rahmen des Projekts EfKom angestrebt, bspw. dringende Aufträge an die schnellsten, zerbrechliche Aufträge an die sorgfältigsten Kommissionierer zu vergeben. Auch die Möglichkeit zur Vermeidung von einseitiger Belastung in Form von bspw. der Zurücklegung langer Distanzen oder der Handhabung großer Massen sowie ein konkreter Ansatzpunkt für gezielte Weiterbildungsmaßnahmen sind gegeben.

Um eine optimale Zuordnung der Kommissionierer unter Berücksichtigung ihrer bekannten Stärken und Schwächen zu den unterschiedlichen Kommissionieraufträgen zu ermöglichen, ist eine Kommissionierauftragsklassifizierung erforderlich. Hierzu bilden kommissionierauftragsbezogene eindimensionale Verteilungsanalysen die entsprechende Grundlage dar. Abbildung 4 illustriert die Verteilung der Anzahl an Entnahmen pro Kommissionierauftrag zweier Kommissioniersysteme. 
Anzahl Entnahmen pro Kommissionierauftrag (bereinigt)

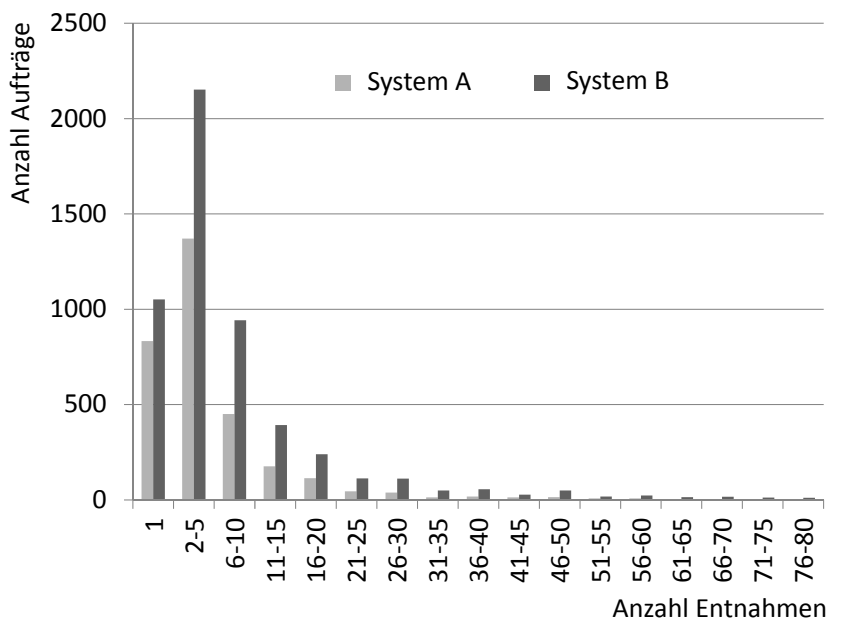

Abbildung 4. Verteilungsanalyse Anzahl Entnahmen pro Kommissionierauftrag

Aus der Häufigkeitsanalyse ergeben sich die Durchschnittswerte (Anzahl Entnahmen pro Kommissionierauftrag) von 7,9 und 10,2 für System A bzw. B. Ähnliche Häufigkeitsanalysen werden für alle Leistungseinflussfaktoren durchgeführt. Die jeweiligen Durchschnittswerte fließen in eine Kommissionierauftrags-Clustering-Methode ein, nach der eingehende Kommissionieraufträge klassifiziert und gruppiert werden. Die Vorgehensweise wird in Abbildung 5 dargestellt.

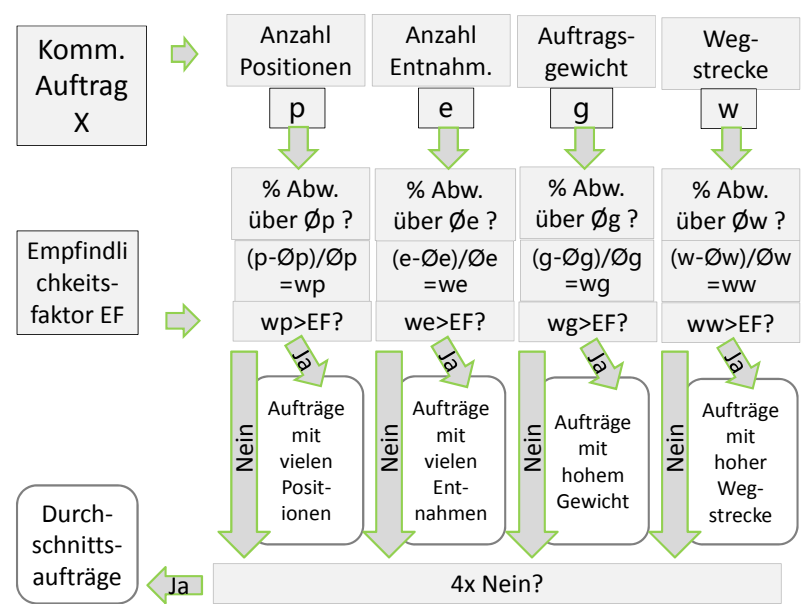

Abbildung 5. Auftragsclustering-Methode unter Berücksichtigung von vier Leistungseinflussfaktoren

Dabei stellt der sogenannte „Empfindlichkeitsfaktor“ eine Variable dar, die bestimmt, bei welcher prozentualen Abweichung das System Aufträge als außerordentlich klassifiziert. Der Einsatz von jeweils einem separat einstellbaren Empfindlichkeitsfaktor pro Leistungseinflussfaktor wäre durchaus denkbar, um eine Berücksichtigung kommissioniersystemspezifischer Besonderheiten zu ermöglichen. Es ergeben sich nach dieser Kommissionierauftragsklassifizierungsmethode grundsätzlich vier unter- schiedliche Kommissionierauftragskonten oder -stapel, die an die passenden Mitarbeiter freigegeben werden können. Die Entwicklung eines entsprechenden Zuordnungsverfahrens sowie dessen Realisierung als EDV-Anwendung stellt eine wesentliche Zielsetzung des Forschungsprojekts dar.

\section{ZUSAMMENFASSUNG UND AUSBLICK}

Viele Einflussgrößen auf die manuelle Kommissionierleistung sind grundsätzlich zwar bekannt, werden jedoch nicht in die Leistungsbewertung integriert und in einem Kennzahlensystem verknüpft. Vor dem Hintergrund der zentralen Bedeutung des Menschen in der Kommissionierung soll im Rahmen des Forschungsprojekts EfKom dieser Umstand beseitigt werden.

Aus arbeitswissenschaftlicher sowie aus finanzieller Sicht sind die Vorteile einer Methode zur ganzheitlichen Kommissionierleistungsbewertung ersichtlich. Die mit ihnen verbundenen Möglichkeiten zur Optimierung sowohl der Personaleinsatzplanung als auch der Auftragssteuerung sind eindeutig. Trotz der weiten Verbreitung manueller Kommissioniersysteme existieren nur unzureichende Ansätze zur Bestimmung der tatsächlich erbrachten Leistung von Kommissionierern. Daher wird im Rahmen des Forschungsprojekts EfKom die Entwicklung einer hinreichend praktikablen Methode zur Individualbzw. Teambewertung von Kommissionierern angestrebt, die primär die operative Personaleinsatzplanung (individuell und in Teams) und sekundär die strategische Kommissioniersystemplanung unterstützt.

Aus der Sicht der an dem Forschungsprojekt EfKom beteiligten Unternehmen (vorwiegend kleine und mittlere Unternehmen) stellt das Projekt eine Chance zur Effizienzsteigerung dar. Die Fähigkeit, individuelle Mitarbeiterleistungen ganzheitlich zu bewerten sowie die Möglichkeit, Kommissionieraufträge den Kommissionierern anhand ihrer unterschiedlichen Stärken und Schwächen gezielt zuzuordnen, impliziert auch eine Leistungssteigerung durch den effizienteren Einsatz des Personals, was sich positiv auf den Unternehmenserfolg auswirkt. Die zur Umsetzung des Projekts benötigten Prozesszeitanalysen dienen zudem der Identifizierung unnötiger Tätigkeiten bei der Kommissionierung sowie übermäßiger Körperbelastung bzw. mangelhafter Arbeitsplatzgestaltung. Durch Verbesserungen der Ergonomie soll der wertschöpfende Anteil der Kommissioniertätigkeiten - bei gleichzeitiger Reduktion der körperlichen Belastung der Mitarbeiter gesteigert werden. Eine erhöhte Wertschöpfung in der Kommissionierung spart Kosten ein und wirkt sich positiv auf die Motivation der Mitarbeiter aus. Unter Nutzung der in den Stärken-Schwächen-Profilen der einzelnen Mitarbeiter hinterlegten Informationen können entsprechende Ansatzpunkte sachlich dargestellt werden, auf deren Basis gezielte Weiterbildungsmaßnahmen planbar sind. 


\section{LITERATUR}

[VDI94]

[Cro07] Crostack, Horst-Artur et al.: AiFForschungsvorhaben Nr.14368 „Optimierung von Kommissionierung und Verpackung durch geeignete Strategien für die Qualitätsprüfung unter Berücksichtigung der Retourenabwicklung (QuinKom)“. Universität Dortmund, $2005-2007$

[Deu09] Deuse, Jochen et al.: AiF- Forschungsvorhaben Nr. 15359 "Skalierbare Kommissioniersysteme - Entwicklung eines Instrumentariums zur Auswahl eines kostenoptimalen skalierbaren Kommissioniersystems unter Berücksichtigung prognostizierter Auslastungsschwankungen “. Universität Dortmund, 2007 - 2009

[GaW10] Galka, Stefan; Günthner, Willibald A.: „Analytische Modellierung von Kommissioniersystemen zur systematischen Planungsunterstützung “. In: Subbert, Sindy; Meynarts, Peter: Tagungsband zum 4. Fachkolloquium der Wissenschaftlichen Gesellschaft für Technische Logistik e. V., Chemnitz, 2008

[Gro83] Großeschallau, Werner: Materialflussrechnung - Logistik in Industrie, Handel und Dienstleistungen. Berlin: Springer-Verlag, 1983

[Gud05] Gudehus, Timm: Logistik - Grundlagen, Strategien, Anwendungen, 3. Auflage. Berlin: Springer-Verlang, 2005

[Hom10] ten Hompel, Michael et al.: AiFForschungsvorhaben Nr. 15811 „Strategien für die flexible auftragsweise Kommissionierung mit integrierter Prüfung mit dem Ziel einer hohen Kapazitätsauslastung eingesetzter Ressourcen (FlexKom)“. Universität Dortmund, $2008-2010$

[Spa09] Spath, Dieter (Hrsg.); Wehking, KarlHeinz (Hrsg.): Der Mensch in der Intralogistik - Ein Handbuch für Führungskräfte. Endbericht des MWKForschungsprojekts „Der Mensch als steuernder und operativer Leistungsträger in der Intralogistik (MensoLIn)“, Universität Stuttgart, 2007 - 2009
Verein Deutscher Ingenieure e.V.: VDIRichtlinie 3590 - Kommissioniersysteme / Grundlagen (Blatt 1). In: VDIHandbuch Material und Fördertechnik, Band 8, VDI-Gesellschaft Fördertechnik Materialfluss - Ausschuss Integrierte Material- und Datenflusssysteme, Berlin: Beuth-Verlag, 1994 